\title{
Diagnostic accuracy of intraoperative consultation of ovarian neoplasms
}

\author{
L. Avesi, N. Kayani, S. Hassan, R. Shahid \\ Department of Pathology and Microbiology, Aga Khan University Hospital, Karachi, Pakistan
}

\section{A B S T R A C T}

Introduction: Ovarian neoplasm's are an important cause of morbidity and mortality in women. Frozen section is widely used in the intra-operative assessment of patients with ovarian tumors to provide guidance for an appropriate surgical management. It is crucial to know intra-operatively, the exact nature of the tumor to determine the extent of surgery. It is in these situations where the Intraoperative frozen section technique plays its role in making this distinction. Methodology: Females of all ages undergoing planned surgeries for ovarian tumors at Aga Khan Hospital or outside referrals with request for intra operative diagnosis was included. Age of the patient, duration of the lesion and clinical impression were recorded and frozen section was performed. Results: Thirty four cases of ovarian tumors received for frozen section were included in this study. The calculated sensitivity was $94.4 \%$, specificity was $93.8 \%$, positive predictive value was $94.4 \%$ and negative predictive value was $93.8 \%$. Conclusion: This study shows that frozen section is a highly sensitive and specific method for the rapid diagnosis of ovarian tumors.

Key words: Frozen section, Ovarian tumors, Accuracy, Diagnosis, Management, Gross examination

\section{INTRODUCTION}

Ovarian neoplasm's are an important cause of morbidity and mortality in women. ${ }^{1}$ Ovarian malignancy accounts for almost $25 \%$ of gynecological cancers and $50 \%$ of all deaths from cancers of the female genital tract. ${ }^{2}$ Diagnosis of an adnexal mass starts with an accurate history and physical examination, with para-clinical study parameters, including sonography, CT scanning, CA125 levels and intraoperative histologic diagnosis (frozen section) being imperative in performing an appropriate operation. ${ }^{3}$

Frozen section is widely used in the intra-operative assessment of patients with ovarian tumors to provide guidance for an appropriate surgical management. The diagnosis of malignancy is usually straightforward, but in some cases it may be difficult to distinguish whether the malignant tumor is of ovarian origin or represents metastases from other sites. ${ }^{4,5}$ It is crucial to know intraoperatively, the nature of the tumor to determine the extent of surgery. It is in these situations where the Intraoperative frozen section technique plays its role in making this distinction and to decide the exact surgical course, where preoperative imaging and tumor markers have only limited value in differentiating the exact tumor origin. The frozen section has been reported to have a good diagnostic accuracy in benign and malignant tumors, but a lower accuracy for borderline tumors. ${ }^{6}$ Generally, the false negative rate is low and the false positive rate is negligible for benign and malignant ovarian tumors, making the frozen section an accurate technique for the histologic diagnosis of ovarian tumors. Deferred diagnoses or incompatible frozen section diagnosis is usually due to technical limitations, especially for the larger specimens of mucinous ovarian tumors, as they require extensive sampling. ${ }^{7}$

Regular re-evaluation or consultation concerning disagreements between frozen section diagnosis and final permanent paraffin diagnosis should be conducted by both surgeons and pathologists as part of quality assurance to 
determine the most appropriate intraoperative management of patients with ovarian tumors. ${ }^{8}$

The real aim of the frozen section procedure is to influence the course of operation and intraoperative management of the patient. Sometimes, it is difficult for the pathologists to render a definite diagnosis on the basis of frozen section, and have to wait for the permanent sections. The results may either be concordant, discordant or deffered. ${ }^{9}$

The rationale of the current study is that if the diagnostic accuracy of intraoperative frozen section is found to be accurate, then it can be used as one piece of evidence for surgeons to use in determining the surgical course for benign and malignant tumors.

\section{MATERIALS AND METHODS}

Females of all age groups undergoing planned surgeries for ovarian tumors at Aga Khan University Hospital (AKUH) or outside referrals with request for intra-operative diagnosis were included in this study. Age of the patient, duration of the mass and clinical impression was documented for each case on a Performa. Gross examination along with frozen section was performed. The number of sections submitted for frozen were 1 to 4 , depending upon the type and size of the tumor. These sections were cut in the cryostat machine. Cryostat is now routinely being used because of the technical excellence of the sections obtained. The sections were further stained with hematoxylin-eosin. All the sections were studied microscopically under low and high power by an experienced pathologist. The frozen section findings and diagnosis was conveyed to the surgical team who then proceeded with the appropriate surgery. The average time taken for the entire procedure (after specimen being received) was approximately 15 minutes. After the frozen section sampling, the remaining specimen was fixed in $10 \%$ buffered formalin, over-night, after which additional sampling was done, from the representative areas. Histologic findings of the frozen section were compared with the final paraffin section diagnosis of the same specimen.

\section{STATISTICS}

Age

The age range in this study was 57 years, with the youngest patient being 18 years of age and oldest being 75 years. The mean age was 40.29 years with a standard deviation of 17.6.

\section{RESULTS}

Thirty four cases of ovarian tumors received for frozen section at Aga Khan University Hospital were taken in this study. The frozen section and permanent section reports were then compared taking the permanent section diagnosis as the gold standard. Two cases fell in the discordant category, while 32 cases showed same results on frozen section as well as on permanent section diagnosis. Mean \pm SD will be computed for age. Sensitivity, specificity, positive and negative predictive value and accuracy will be calculated for frozen section of benign and malignant ovarian tumors by taking paraffin embedded sections as the gold standard. Confidence interval $95 \%$ will be calculated for sensitivity, specificity, positive and negative predictive value and accuracy for benign and malignant tumors, and shown in Table 1.

The results of the frozen section diagnosis are shown in Table 2.

The results of the permanent section diagnosis with their frequencies and percentages are shown in Table 3.

\section{DISCUSSION}

Ovarian tumors affect a significant number of the female population. They are not always malignant, but the feeling of harboring any tumor by an individual is quite fearsome. Malignant ovarian neoplasm's are responsible for $4 \%$ of all cancers effecting women and are the second most common cause of death from gynecological cancer and fourth most common cause of death from all types of cancers effecting women. ${ }^{10}$

\section{Table 1: Statical results}

\begin{tabular}{ll} 
Sensitivity & $94.4 \%$ \\
Specificity & $93.8 \%$ \\
Positive predictive value & $94.4 \%$ \\
Negative predictive value & $93.8 \%$ \\
\hline
\end{tabular}

\begin{tabular}{lcl}
\multicolumn{3}{l}{ Table 2: Frozen section diagnosis } \\
\hline $\begin{array}{l}\text { Serial } \\
\text { no: }\end{array}$ & $\begin{array}{l}\text { No: of } \\
\text { cases }\end{array}$ & Diagnosis on frozen section \\
\hline 1. & 12 & Benign \\
2. & 5 & Benign Cyst \\
3. & 3 & Mature Cystic Teratoma \\
4. & 2 & Benign Mucinous Neoplasm \\
5. & 1 & Serous Surface Epithelial Neoplasm \\
6. & 1 & Neoplastic Lesion \\
7. & 1 & Spindle Cell Lesion \\
8. & 1 & Stromal Tumour \\
9. & 1 & Inflammation and Infection \\
10. & 1 & Benign Cyst with Focal Mucinous Areas \\
11. & 1 & Adenocarcinoma \\
12. & 1 & Ovarian Carcinoma \\
13. & 2 & Malignant Neoplasm probably Carcinoma \\
14. & 1 & Malignant Neoplasm most likely Carcinoma \\
15. & 1 & Malignant Epithelial Neoplasm \\
\hline
\end{tabular}

Asian Journal of Medical Sciences | Jan-Mar 2015 | Vol 6 | Issue 1 


\begin{tabular}{|c|c|c|c|c|}
\hline Diagnosis & Frequency & Percent & $\begin{array}{l}\text { Valid } \\
\text { percent }\end{array}$ & $\begin{array}{c}\text { Cumulative } \\
\text { percent }\end{array}$ \\
\hline $\begin{array}{l}\text { Acute and Chronic } \\
\text { Inflammation }\end{array}$ & 1 & 2.9 & 2.9 & 2.9 \\
\hline Adult granulose cell & 1 & 2.9 & 2.9 & 5.9 \\
\hline \multicolumn{5}{|l|}{ Tumor } \\
\hline Benign cystic lesion & 1 & 2.9 & 2.9 & 8.8 \\
\hline Benign mucinous & 2 & 5.9 & 5.9 & 14.7 \\
\hline \multicolumn{5}{|l|}{ Cystadenoma } \\
\hline Benign serous cyst & 1 & 2.9 & 2.9 & 17.6 \\
\hline Benign serous & 1 & 2.9 & 2.9 & 20.6 \\
\hline \multicolumn{5}{|l|}{ Cystadenofibroma } \\
\hline Benign serous & 2 & 5.9 & 5.9 & 26.5 \\
\hline \multicolumn{5}{|l|}{ Cystadenoma } \\
\hline Bilateral mature cystic & 1 & 2.9 & 2.9 & 29.4 \\
\hline \multicolumn{5}{|l|}{ Teratoma } \\
\hline Bilateral mucinous & 1 & 2.9 & 2.9 & 32.4 \\
\hline \multicolumn{5}{|l|}{ Cystadenoma } \\
\hline Clear cell carcinoma & 2 & 5.9 & 5.9 & 38.2 \\
\hline Endometriotic cyst & 2 & 5.9 & 5.9 & 44.1 \\
\hline Endometriotic & 2 & 5.9 & 5.9 & 50.0 \\
\hline \multicolumn{5}{|l|}{ Adenocarcianoma } \\
\hline Endometriotic cyst & 1 & 2.9 & 2.9 & 52.9 \\
\hline Endometriotic ovarian & 1 & 2.9 & 2.9 & 55.9 \\
\hline \multicolumn{5}{|l|}{ Cyst } \\
\hline Granulosa cell tumor & 1 & 2.9 & 2.9 & 58.8 \\
\hline $\begin{array}{l}\text { Mature cystic } \\
\text { teratoma }\end{array}$ & 6 & 17.6 & 17.6 & 76.5 \\
\hline $\begin{array}{l}\text { Mucinous } \\
\text { cystadenoma }\end{array}$ & 3 & 8.8 & 8.8 & 85.3 \\
\hline Poorly differenciated & 1 & 2.9 & 2.9 & 88.2 \\
\hline \multicolumn{5}{|l|}{ Carcinoma } \\
\hline Serous cystadenoma & 2 & 5.9 & 5.9 & 94.1 \\
\hline Tuboovarianabcess & 1 & 2.9 & 2.9 & 97.1 \\
\hline Yolk sac tumour & 1 & 2.9 & 2.9 & 100.0 \\
\hline Total & 34 & 100.0 & 100.0 & \\
\hline
\end{tabular}

The overall risk of malignancy in ovarian neoplasm's is $13 \%$ in premenopausal, and $45 \%$ in postmenopausal women. ${ }^{3}$ Among all types of ovarian tumors registered, the incidence of malignancy is about $15-20 \%$ in different parts of world. These tumors behave in a diverse way and generally escape detection until they attain a larger size. ${ }^{11,12}$ Clinically detected ovarian masses have a heterogenous nature, including neoplasm's and benign non neoplastic lesions or cysts. Primary ovarian neoplasm's form a complex group of tumors that exhibit a wide variety of histological features and are classified according to the histogenetic principles set by World Health Organization (WHO) and this classification categorizes ovarian tumors with regard to their derivation from coelomic surface epithelial cells, germ cells, and mesenchyme (the stroma and the sex cord). ${ }^{13}$ Furthermore, patients with ovarian metastasis from nongenital regions may have a presentation similar to primary ovarian cancer, which can contribute to confusion in the diagnosis and inappropriate management. ${ }^{14}$

Frozen section technique, introduced by William Welsh in $1891,{ }^{3}$ is used in both gynecological and general surgical practice mainly to aid in the differentiation of benign from malignant disease. The accuracy of frozen section diagnosis for the assessment of ovarian masses is good, with acceptable sensitivities for almost perfect specificities. ${ }^{15}$ It is one of the most important and difficult procedures that pathologists performs during their practice. Its three legitimate purposes are,

(1) To establish the presence and nature of lesions

(2) To determine the adequacy of surgical margins

(3) To establish whether the tissue obtained contains diagnosable material or whether additional sampling is indicated.

Ovarian tumors of borderline malignancy are also an important group, characterized by some histologic features of malignancy (epithelial cell stratification, increased mitotic activity, nuclear atypia). The overall sensitivity of frozen section for benign and malignant lesions varies from $65 \%$ to $97 \%$ and $71 \%$ to $100 \%$ respectively. The overall sensitivity and specificity for borderline tumors is $45.4 \%$ and $98.3 \%$, respectively. ${ }^{1}$

Various reasons have been advanced for the relative inaccuracy of frozen section in the diagnosis of borderline tumors. In a large borderline tumor, there may be only a few foci of frank malignancy that may require a large number of frozen section samples for diagnosis. This is very labor intensive and usually beyond the capabilities of most laboratories. ${ }^{6}$ Since the accuracy rate for borderline ovarian tumors is low, care and attention is required for improvement in this field. ${ }^{16,17}$ Preoperative evaluation of patients with an ovarian mass is usually made by clinical assessment, imaging studies and determination of serum levels of tumor markers. Since these methods have limited value for recognition of ovarian malignancy, this method can assist the surgeon to tailor the extent of surgery.

This diagnostic procedure could reduce the possibilities of doing incomplete surgery for benign tumors (oophorectomy or cystectomy) or radical surgery for malignant conditions, in a majority of patients. Frozen section is sometimes referred to as intraoperative consultation, as its practice usually involves a liaison between the surgeon and gynecological pathologist, rather than the mere provision of a histological diagnosis. Accuracy of frozen section depends on a number of factors such as the number of specimens processed, number of slices of specimen analyzed and nature of tissue. Of note is that the report of benign histology on frozen section is only a report of that percentage of tissue analyzed. ${ }^{18}$ Therefore, intraoperative frozen section is necessary, both as a quick histologic diagnosis and as a guide for the surgeons in planning an appropriate management. 
Our study results showed a sensitivity of $94.4 \%$, and a specificity of $93.8 \%$. The positive predictive value was $94.4 \%$ and the negative predictive value was $93.8 \%$. These results are comparable to the results of a study by Washington et al, ${ }^{19}$ in which 376 cases of ovarian tumors were taken and their frozen section and permanent section results were compared. The calculated sensitivity of this was $98.2 \%$ and specificity was $88.6 \%$. The positive predictive value was $92.2 \%$ and negative predictive value was $97.2 \%$.

Another study by Rakhshan et al, ${ }^{20}$ that included 282 ovarian masses and submitted them for frozen section. The results of this study were also similar to ours. The calculated sensitivity was $92 \%$ to $99 \%$ and the calculated specificity was $91 \%$ to $99 \%$.

Pinto et al, ${ }^{21}$ conducted a study on 243 ovarian biopsies received for frozen section and the results showed 93\% sensitivity and $99 \%$ specificity, similar to our study.

\section{CONCLUSION}

The study shows that frozen section is a highly sensitive and specific method for the rapid diagnosis of ovarian tumors, with calculated sensitivity (94.4\%), specificity $(93.8 \%)$ positive predictive value $(94.4 \%)$ and negative predictive value $(93.8 \%)$.

\section{ACKNOWLEDGEMENT}

We thank the Management and the Chair person of the Department of Pathology and Microbiology Aga Khan University Hospital to have permitted and supported this project. We are also grateful to Dr. Naila Kayani Professor of Pathology for her supervision.

\section{REFERENCES}

1. Maheshwari A, Gupta S, Kane S, Kulkarni Y, Goyal BK and Tongaonkar HB. Accuracy of intraoperative frozen section in the diagnosis of ovarian neoplasms. Experience at a tertiary oncology center. World J Surg Oncol 2006; 24:12-16.

2. Naheed I, Malik S and Shaukat MS. Review of ovarian tumors. Ann King Edward Med Coll 2001;7:180-182.

3. Ghaemmaghami F, Fakour F, Karimi Zarchi M, Behtash N, Modares Gilani M, Mousavi A, et al. Clinical assessment, gross examination, frozen section of ovarian masses: do patients benefit? Arch Gynecol Obstet 2008; 278:209-213.
4. Stewart CJ, Brennan BA, Hammond IG, Leung YC and McCartney AJ. Accuracy of frozen section in distinguishing primary ovarian neoplasia from tumors metastatic to the ovary. Int J Gynecol Pathol 2005;24:356-62.

5. Stewart CJ, Brennan BA, Hammond IG, Leung YC and McCartney AJ. Intraoperative assessment of ovarian tumors. A 5-year review with assessment of discrepant diagnostic cases. Int J Gynecol Pathot 2006;25:216-222.

6. Moodley $M$ and Bramdev A. Frozen section. Its role in gynaecological oncology. J Obstet Gynaecol 2005;25:629-634.

7. Ivanov $S$ and Khadzhiolov N. Ovarian tumours: accuracy of frozen section diagnosis. Akush Ginekol (Sofiia) 2005;44:11-13.

8. Geomini P, Bremer G, Kruitwagen R and Mol BW. Diagnostic accuracy of frozen section diagnosis of the adnexal mass. a meta-analysis. Gynecol Oncol 2005;96:1-9.

9. Robert E. Scully, Robert H. Young and Philip B. Clement. Tumors of the Ovary, Maldeveloped Gonads, Fallopian Tube and Broad Ligament. The Journal of Pathology 1999; 189: 1-146.

10. Medeiros L, Rosa D, Edelweiss M, Stein AT, Bozzetti MC, Zelmanowicz A, et al. Accuracy of frozen-section analysis in the diagnosis of ovarian tumors. a systematic quantitive review. International Journal of Gynecological Cancer 2005; 15: 192-202.

11. Shaikh NA, Hashmi F, Samoo RP. Pattern of Ovarian Tumors: Report of 15 years experience at Liaquat University Jamshoro. JLUMHS 2007;13-5.

12. Vaidya $S$, Sharma $P, K C S$, Vaidya $S A$. Spectrum of ovarian tumors in a referral hospital in Nepal. Journal of Pathology of Nepal 2014; 4: 539-543.

13. Kaku T, Ogawa S, Kawano Y Ohishi Y, Kobayashi H, Hirakawa T, et al. Histological classifications of ovarian cancer. Medical Electron Microscopy 2003; 36:9-17.

14. Suprasert, Khunamornpong S, Phusong A,Settakorn Jand Siriaungkul S. Accuracy of Intra-operative frozen sections in the diagnosis of ovarian masses. Asian Pacific Journal of Cancer Prevention 2008;9:737-740.

15. Rosai J. Rosai and ackerman's surgical pathology. 9th ed. Elsevier; 2004.

16. Wootipoom V, Dechsukhum C, Hanprasertpong J andLim A. Accuracy of intraoperative frozen section in diagnosis of ovarian tumors. J Med Assoc Thai 2006;89:577-582.

17. Subbian A, Devi UK, Bafna UD. Accuracy rate of frozen section studies in ovarian cancers: A regional cancer institute experience. Indian J Cancer 2013;50:302-5.

18. Ghaemmaghami F, Behnamfar F and Ensani F. Intraoperative Frozen Sections for Assessment of Female Cancers. Asian Pacific Journal of Cancer Prevention 2007; 8:635-639.

19. Wasinghon $P$, Suthippintawong $C$ and Tuipae $S$. The accuracy of intraoperative frozen sections in the diagnosis of ovarian tumours. Journal Med Assoc Thai 2008; 91:1791-1795.

20. Rakhshan A, Zham $\mathrm{H}$ and Kazempour M. Accuracy of frozen section diagnosis in ovarian masses. Experience at a tertiary oncology center. Arch GynecolObstet 2009;280:223-228.

21. Pinto $P$, Andradre $L$ and Derchain $S$. Accuracy of intraoperative frozen section diagnosis of ovarian tumours. Gynecologic Oncology 2001;81:230-233.

Source of Support: Nil, Conflict of Interest: None declared. 